\title{
KurSL: model of anharmonic coupled oscillations based on Kuramoto coupling and Sturm-Liouville problem
}

Article

Accepted Version

Laszuk, D., Cadenas, J. O. and Nasuto, S. J. (2018) KurSL: model of anharmonic coupled oscillations based on Kuramoto coupling and Sturm-Liouville problem. Advances in Data Science and Adaptive Analysis, 10 (02). p. 1840002. ISSN 2424-922X doi: https://doi.org/10.1142/s2424922x18400028 Available at https://centaur.reading.ac.uk/78829/

It is advisable to refer to the publisher's version if you intend to cite from the work. See Guidance on citing.

To link to this article DOI: http://dx.doi.org/10.1142/s2424922x18400028

Publisher: World Scientific Publishing

All outputs in CentAUR are protected by Intellectual Property Rights law, including copyright law. Copyright and IPR is retained by the creators or other copyright holders. Terms and conditions for use of this material are defined in the End User Agreement.

www.reading.ac.uk/centaur 
Central Archive at the University of Reading

Reading's research outputs online 
Advances in Data Science and Adaptive Analysis

(C) World Scientific Publishing Company

\title{
KurSL: Model of anharmonic coupled oscillations based on Kuramoto coupling and Sturm-Liouville problem
}

\author{
Dawid Laszuk $^{*}, \ddagger$, Oswaldo Cadenas ${ }^{\dagger, \S}$ and Slawomir J. Nasuto*, ${ }^{*}$ \\ ${ }^{*}$ School of Biological Sciences, University of Reading, \\ Reading, RG6 6AY, United Kingdom \\ School of Engineering, London South Bank University, \\ London, SE1 OAA, United Kingdom \\ laszukdawid@gmail.com \\ cadenaso@lsbu.ac.uk \\ s.j.nasuto@reading.ac.uk \\ Received 11 March 2018 \\ Revised 9 May 2018 \\ Published
}

\begin{abstract}
Physiological signalling is often oscillatory and shows nonlinearity due to complex interactions of underlying processes or signal propagation delays. This is particularly evident in case of brain activity which is subject to various feedback loop interactions between different brain structures, that coordinate their activity to support normal function. In order to understand such signalling in health and disease, methods are needed that can deal with such complex oscillatory phenomena. In this paper, a data-driven method for analysing anharmonic oscillations is introduced. The KurSL model incorporates two well-studied components, which in the past have been used separately to analyse oscillatory behaviour. The Sturm-Liouville equations describe a form of a general oscillation, and the Kuramoto coupling model represents a set of oscillators interacting in the phase domain. Integration of these components provides a flexible framework for capturing complex interactions of oscillatory processes of more general form than the most commonly used harmonic oscillators. The paper introduces a mathematical framework of the KurSL model and analyses its behaviour for a variety of parameter ranges. The significance of the model follows from its ability to provide information about coupled oscillators' phase dynamics directly from the time series. KurSL offers a novel framework for analysing a wide range of complex oscillatory behaviours, such as encountered in physiological signals.
\end{abstract}

Keywords: Kuramoto; Sturm-Liouville; Oscillation; Coupling. 


\section{Introduction}

Modelling and analysis of oscillations are of prime interest in the natural sciences due to the abundance of such phenomena in nature [Winfree, 1967] Examples of physiological oscillatory processes range from the circadian rhythm, through synchronisation of fireflies flashing to the behaviour of the main constituents of the cardiovascular system [Strogatz, 2000; Acebrón et al., 2005]. Moreover, technologies used for recording of physiological signals, such as magnetic resonance imaging (MRI), also utilise various oscillatory effects [Acebrón et al., 2005]. A common assumption when analysing such systems is that they are isolated from their surroundings and that their behaviour can be described in terms of harmonic oscillators or their linear combinations. For this reason, Fourier series has been a popular and powerful tool for describing oscillatory phenomena. In case of relatively simple systems conforming to such assumptions, the method's usage has been sufficient to provide a meaningful description of systems' behaviour and has underpinned many advances in applied mathematics, physics and engineering. Other popular methods, e.g. wavelet transform, try to describe a more general form of oscillations; however, they again assume the independence and linearity of the underlying components.

However, not all oscillations found in nature are sinusoidal, or equivalently, it is not always the case that harmonic oscillators can adequately describe the mechanism of generating oscillations. More complex systems require a different approach, as the periodicity of a system does not necessarily imply that it will repeat the same behaviour over time. The perturbation to its state may be due to outside influences, and their effect will vary depending on the scale, at which they are analysed. Even a relatively simple system, such as a pendulum, may be described as harmonic oscillator only up to a first approximation. Its movement is subject to perturbations such as friction, air drag or the fact that the force causing the movement around equilibrium is not linearly proportional to displacement for large displacement angles. Such cases are called anharmonic, which signifies that their potential energy cannot be locally described as a quadratic function and thus the resulting movement is not harmonic.

Several methods commonly used to extract information from anharmonic oscillatory systems can only be meaningfully applied under restrictive and simplified assumptions [Cohen, 1995; Cohen and Gulbinaite, 2013]. Many natural systems are non-linear and non-stationary, with all component properties, such as instantaneous frequency varying in time [Boashash, 1992]. These properties are not merely analytic inconveniences, but potential carriers of meaningful information about systems' behaviour or function, manifesting in subtle interactions within them, as well as between the systems and their surroundings [Galka, 2000]. Methods mentioned above have not been designed to capture such phenomena and may miss essential characteristics necessary to understand system's operation.

Increasing appreciation of such shortcomings has led to attempts by several researchers to mitigate them by proposing data-driven methods [Huang et al., 1998; 
Duggento et al., 2012]. An example of such a method is the empirical mode decomposition (EMD) [Huang et al., 1998]. EMD iteratively decomposes a signal into a set of oscillations of a general form. EMD has been proven to provide useful information about systems containing oscillations. For example, in biomedical studies, it has shown great promise in characterising the synchronisation patterns correlates of mild cognitive decline in electroencephalograms [Sweeney et al., 2013; SweeneyReed and Nasuto, 2007, 2009], non-linear filtering of the electromyograms [Andrade et al., 2006, 2008], analysis of tremor [de Lima et al., 2006], or detecting eye movements [Rutkowski et al., 2010; Molla et al., 2010]. However, due to its empirical behaviour, it is difficult to understand the operation of the method and the consequences of choices made during the intermediate steps of the iterative decomposition process and their effects on the resulting components. Given increasing interest in understanding the complex oscillatory phenomena, there is a great need for the development of techniques aimed at general anharmonic oscillations analysis that share the flexibility of data-driven techniques such as EMD, yet are proposed within a more principled mathematical framework, thus enabling their better understanding and analysis [Sharpley and Vatchev, 2006; Chu et al., 2013; Laszuk et al., 2016].

The paper proposes a forward model that can be used as a mathematical framework for decomposition method, which would fill the gap mentioned above. The model assumes that complex oscillations arise in systems with many interacting oscillatory components. Such interactions can result in oscillations which have nonharmonic behaviour concomitant to amplitude- and phase-modulations. Thus, the proposed approach is based on a synthesis of two ubiquitous models of oscillatory phenomena: the Sturm-Liouville eigenproblem and the Kuramoto coupling model. The former is an ordinary differential equation describing a general form of oscillations, which has been widely utilised in modelling of physical phenomena, including biomedical areas such as modelling circulatory system [Pontrelli and de Monte, 2009; Gou and Chen, 2015], biophysical signal separation [Singer, 2006] and propagation of electromagnetic waves [Moran et al., 2007; Carvalhaes and de Barros, 2015]. The second component of the proposed method, the Kuramoto model, provides means of capturing how a set of oscillations could interact with each other [Acebrón et al., 2005]. This model has been successfully applied in a variety of fields describing coupled oscillatory systems [Brown et al., 2003; Acebrón et al., 2005; Rodrigues et al., 2016], including biological systems, such as cortical activity [Breakspear et al., 2010; Tauro et al., 2014; Sadilek and Thurner, 2015].

The outline of this paper is as follows. The second section introduces aforementioned models of oscillatory phenomena. Then, Section 3 presents how an integration of the models defined in the previous section produces the KurSL model and its components. In Section 4, a number of experiments have been performed that analyse properties of the method's mapping from parameter space onto time series, and phase dynamics spaces. The document finishes with a conclusion Section 5 about the model and its potential applications. 


\section{Theoretical background}

\section{Sturm-Liouville equation}

The mathematical definition of an oscillation can be formulated in terms of a solution of a second order differential equation (ODE). Its particular form was named after Jacques Charles François Sturm [Sturm, 1836] and Joseph Liouville [Freiling and Yurko, 2001]. Their research focused on ODEs of the form

$$
-\frac{d}{d t}\left(p(t) \frac{d y(t)}{d t}\right)+q(t) y(t)=\lambda w(t) y(t)
$$

where coefficient functions $p(t), q(t)$ and derivative $p^{\prime}(t)$ belong to Hilbert space $\mathcal{L}^{2}$. Equation (1) is in fact an eigenvalue problem with $\lambda$ and $w(t)$ being an eigenvalue and weighting function, respectively. Specific forms of the general definition above have been widely used to represent different oscillatory phenomena. Often, in practice, a simplified form of the equation is used [Freiling and Yurko, 2001], i.e.

$$
y^{\prime \prime}(t)+Q(t) y(t)=\lambda y(t)
$$

where $Q(t)$ is a potential function governing the behaviour of the system. Such representation is especially popular when analysing inverse Sturm-Liouville (SL) problem [Freiling and Yurko, 2001]. Examples of where the SL is used include wave propagation in materials like strings or drums [Halliday et al., 2000], or dynamics of particles in quantum mechanics [Schrödinger, 1926].

Properties and solutions for some particular coefficient functions, i.e. $Q(t)$ or $\{p(t), q(t)\}$, have already been intensively studied. Two of the most popular equations are Bessel type with $\left(p(t)=-t, q(t)=\left(t^{2}-\nu^{2}\right)\right)$ and Airy $(p(t)=-1$, $q(t)=-t)$ [Teschl, 2012]. Solutions to such equations are special functions, which often appear in quantum mechanics [Landau and Lifshitz, 1965]. Another special case of SL equations with published in-depth analysis is one with a constant potential function, $Q(t)=0$. In this case, often called Fourier type, equation (2) is transformed into

$$
y^{\prime \prime}(t)=\lambda y(t)
$$

which for $\lambda<0$ is solved by sinusoids with period of $T=2 \pi / \sqrt{|\lambda|}$, i.e. $y(t)=$ $A \sin (\sqrt{|\lambda|} t+\phi)$.

\section{Kuramoto coupling}

Complex systems can be defined as those comprised of many mutually interacting components. These interactions are the crucial characteristic of system's complexity and different approaches can be taken to describe them. For example, a large system comprising of many uncoupled components can be studied in a reductive manner with each component analysed separately. However, in case when they are coupled, such approach might not be suitable since a strong and persistent coupling between a few components can mimic a behaviour of a single large uncoupled system. The 
complex behaviour emerges from a more subtle network of waxing and waning interactions which in turn can affect dynamics of all components. In case of oscillators these changes could be manifested as periodic perturbations [Strogatz, 2000]. Such interactions between objects have been observed [Strogatz, 1994] in many physical systems, including a cardiorespiratory system [Michaels et al., 1987; Rosenblum et al., 2002] and neural-networks [Galán et al., 2005; Herzog, 2007]. Even in case of the brain, studies of EEG signals [Schnitzler and Gross, 2005; Osterhage et al., 2008; Wagner et al., 2010] suggest that the brain's behaviour could be a result of complex interactions between its regions. A common approach when analysing periodic systems is to consider their dynamics in the phase space, $\Phi$, as

$$
\dot{\phi}_{i}=\omega_{i}+C(\vec{\phi}, t) \text {, }
$$

where each oscillator can be coupled with others by a coupling function $C(\vec{\phi}, t)$ that is dependent on the whole system $\vec{\phi}$. The most popular model describing such collective dependencies was proposed by Kuramoto [Kuramoto, 1975]. It represents components of a complex system by identical coupled oscillators, where the coupling function $C(\vec{\phi}, t)$ between all pairs of oscillators has a form of a scaled sine of a relative phase between each pair, i.e.

$$
\dot{\phi}_{i}=\omega_{i}+\frac{K}{N} \sum_{j=1}^{N} \sin \left(\phi_{j}-\phi_{i}\right)
$$

where $\phi_{i}$ is a phase of the $i^{\text {th }}$ oscillator and $\omega_{i}$ is its natural frequency often called intrinsic frequency. This particular structure of the coupling function was inferred from expected interactions between a set of oscillators. Since individual oscillators have periodic behaviour, it is possible to characterise their trajectories using only phase information. The assumption is that with perfectly synchronised oscillators, i.e. no difference between phases, there would be very little additional interaction; the interaction would increase with an increase in phase gap. Moreover, since the phase functions are only affected by the coupling function, for the former to be periodic, the latter also needs to have a periodic structure. The simplest model that fulfils these constraints is the Kuramoto model. Its popularity is both due to it being simple enough to be analytically solvable for a large number of oscillators, yet powerful enough to explain many of physical phenomena with coupled oscillators.

The coupling function $C(\vec{\phi}, t)$, however, may be reflecting more complex interactions between the oscillators which are not necessarily in a periodic form. In such cases, the coupling function can be represented in the form of Fourier series. In the case of a series consisting of $M$ components, one can define a coupling function as follows

$$
K_{M}\left(\phi_{i}, \phi_{j}, t\right)=\sum_{m=1}^{M} k_{m} \sin \left(m\left(\phi_{j}(t)-\phi_{i}(t)\right)\right),
$$


where $k_{m}$ indicates the strength of the $m^{\text {th }}$ harmonic component. The case for $m=0$ is omitted as it refers to no coupling between oscillators, i.e. $K_{0}=0$. Parameter $M$ is referred to as the model's order as it specifies the number of terms used in the coupling function. Since the order enumerates sinusoids, a model of the $M^{\text {th }}$ order includes all models with lower orders. This inclusion means that larger $M$ corresponds to more flexible models. However, in practice, adding extra dimensions to search space may result in a computationally difficult problem or a model overfitting to noise. Selecting appropriate order is essential as it should be large enough to explain the phenomenon in question, but also small enough to be computable and to avoid overfitting.

\section{KurSL}

One of the fundamental aims in defining models is to provide meaning to data. SL equation (sec. 2) focuses on individual oscillators and tries to capture their overall behaviour. It represents a broad range of oscillations, explaining how their amplitudes may change over time. The Kuramoto model, however, is focusing on the idealised view of oscillators represented solely by their phases. It provides a possibility of the quantitative analysis of their dynamics under mutual coupling. Despite being very valuable and used in the literature to explain many phenomena, the importance of both models is mainly theoretical, as it is impossible to observe an isolated oscillator or to measure only phases. Integrating both modelling approaches may offer a natural extension building on their respective strengths. Sturm-Liouville eigenvalue problem introduces all possible forms of functions that have oscillatory property. Unfortunately, the family of solutions is too big to be analysed collectively. Addition of Kuramoto synchronisation model not only gives meaning to solutions but also restricts the number of possible solutions. A combination of both models can decompose multicomponent signals and provide their individual fundamental properties and instantaneous characteristics.

Figure 1 contains a visual representation of a possible complex system of interacting oscillators. It illustrates how despite a complex configuration of mutually interacting oscillators within the system, a single probe can measure only a collective response. To learn about all individual oscillators, one has to construct and fit an appropriate model. We argue that KurSL model is very well suited for describing systems with general oscillators, as it naturally captures scenarios such as the one depicted in Figure 1. In this section, we introduce and discuss the KurSL model.

\subsection{Definition}

One can describe oscillation as a behaviour which repeats itself over time regardless of the actual path that has been covered. Mathematically, this can be defined by a condition $z(t)=z(t+T)$ for all time $t$ and certain period $T$. A general form that fulfils such criterion, expressed in polar coordinates, is $z(t)=r(t) \exp (i \phi(t))$, 


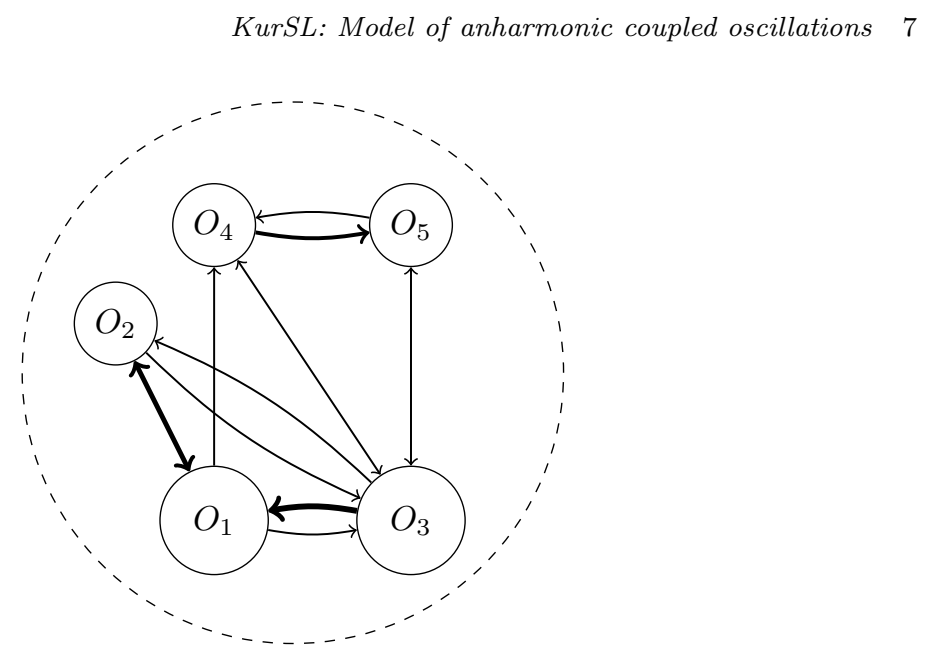

Fig. 1: A graphical example of how oscillators can be connected. Through the direction and the thickness, each arrow represents directionality and magnitude of the interaction between selected oscillators.

which is a product of two periodic functions: an amplitude $r(t)$ and phase-related $\cos \phi(t)$ component. Considering only signals real part, which is measurable, the mathematical definition for oscillation can be written as

$$
y(t)=r(t) \cos (\phi(t)),
$$

which implicitly assumes that the point of reference is within the covered path. The amplitude $r$ and phase $\phi$ can be considered as actual distance from the point of reference and the phase of a cycle. Substituting such a form into the SL equation (2), one obtains

$$
\cos (\phi)\left(\ddot{r}+\left(Q-\lambda-\dot{\phi}^{2}\right) r\right)-\sin (\phi)(2 \dot{r} \dot{\phi}+r \ddot{\phi})=0,
$$

which, due to the mutual instantaneous orthogonality of sine and cosine functions leads to two coupled equations

$$
\left\{\begin{aligned}
2 \dot{r} \dot{\phi}+r \ddot{\phi} & =0, \\
\ddot{r}+\left(Q-\dot{\phi}^{2}\right) r & =0 .
\end{aligned}\right.
$$

These equations describe a relation between amplitude and phase for given function $Q$. For simplicity of notation, in the equation (9) $\lambda$ value was omitted since it can be treated as an offset for the function $Q$.

SL describes a single oscillation which is affected by its environment. In the case when the surroundings consist of other oscillators these interactions can be explicitly modelled. The KurSL model assumes that these interactions are described by the Kuramoto coupling which quantifies how all oscillators within a system affect each other. In its simplest form, where the coupling weights are all equal to $K / N$, the 
relation between phases is introduced as

$$
\dot{\phi}_{i}=\omega_{i}+\frac{K}{N} \sum_{j=1}^{N} \sin \left(\phi_{j}-\phi_{i}\right),
$$

where indices refer to different oscillators from a set of $(i, j \in\{1 . . N\})$. The equation (10) also describes instantaneous frequency of the $i^{\text {th }}$ oscillator, which is defined as a derivative of a phase. Such a system is described by a set of $N$ coupled ODEs and can be fully evaluated knowing $2 N+1$ parameters, i.e. coupling strength $K, N$ intrinsic frequencies $\omega_{i}$ and $N$ initial phases $\phi_{0}$. However, such model assumes mutual, symmetric interaction between all oscillators, which for most physical models might not be true. Introduction of a more general approach to KurSL is done by proposing a flexible form of coupling function $C(\vec{\phi}, t)(4)$, i.e.

$$
C(\vec{\phi}, t)=\sum_{m=1}^{M} \sum_{j=1}^{N} k_{i, j}^{m} \sin \left(m\left(\phi_{j}-\phi_{i}\right)\right),
$$

which highlights a possibility of asymmetric interactions of arbitrary form and admits scenarios, in which some distant oscillators may not affect each other directly. Harmonic components make sure that any type of interaction is included, as per Fourier series theorem any periodic function can be expressed in such a way. However, introducing presented flexibility in coupling model has a significant impact on its complexity. With the generalised form of coupling, the number of parameters increases depending on the order $M$ by $M \cdot N(N-1)$, resulting in a total of $2 N+M \cdot N(N-1)$ parameters for phase coupling model.

Combination of both SL and Kuramoto models leads to the KurSL system that is defined by $3 N$ equations: $N$ for amplitudes $r_{i}$, phases $\phi_{i}$ and potentials $Q_{i}$, respectively,

$$
\begin{aligned}
& \dot{\phi}_{i}=\omega_{i}+C(\vec{\phi}, t), \\
& 2 \dot{r}_{i} \dot{\phi}_{i}+r_{i} \ddot{\phi}_{i}=0, \\
& \ddot{r}_{i}+\left(Q_{i}-\dot{\phi}_{i}^{2}\right) r_{i}=0,
\end{aligned}
$$

where index $i$ indicates that each set is for a single $i^{\text {th }}$ oscillator. In order to explicitly define the KurSL model one needs to assume a type of coupling function $C(\vec{\phi}, t)$ acting within the coupled system. Due to the mutual interactions encapsulated in the Kuramoto model, when considering more than two oscillators, the KurSL model needs to be solved numerically. Simplification of representation and improvement in computation can be obtained by decreasing number of dependent variables by rearranging equations. It can be shown that (13) and (14) can be transformed into 
simpler forms either dependent on the amplitude $r(t)$

$$
\begin{aligned}
\dot{\phi}_{i}(t) & =\frac{r_{i 0}^{2} \dot{\phi}_{i 0}}{r_{i}^{2}(t)}, \\
Q_{i}(t) & =-\frac{\ddot{r}(t)}{r(t)}+\frac{r_{0}^{4} \dot{\phi}_{0}^{2}}{r^{4}(t)},
\end{aligned}
$$

or on the instantaneous frequency $v=\dot{\phi}$,

$$
\begin{aligned}
r_{i}^{2}(t) & =\frac{r_{i 0}^{2} v_{i 0}}{v_{i}(t)}, \\
Q_{i}(t) & =-\frac{\ddot{v}_{i}}{v_{i}}+\frac{3}{4} \frac{\dot{v}_{i}^{2}}{v_{i}^{2}}+v_{i}^{2},
\end{aligned}
$$

wherein both cases $r_{i 0}$ and $\dot{\phi}_{i 0}=v_{i 0}$ are the initial values of the amplitude and the instantaneous frequency, respectively. Since synchronisation in phase cannot be simplified, the canonical representation of the system is dependant on the phase function, i.e.

$$
\begin{aligned}
v_{i} & =\omega_{i}+C(\vec{\phi}, t), \\
Q_{i}(t) & =-\frac{\ddot{v}_{i}}{v_{i}}+\frac{3}{4} \frac{\dot{v}_{i}^{2}}{v_{i}^{2}}+v_{i}^{2} .
\end{aligned}
$$

Such a coupled set of ODEs fully describes the KurSL system. Recalling that each oscillator is composed of an amplitude and a phase-related function (7) one can present component in a form dependent only on the phase, i.e.

$$
y_{i}(t)=r_{i 0} \sqrt{\frac{\dot{\omega}_{i 0}}{\dot{\phi}_{i}(t)}} \cos (\phi(t)) .
$$

This highlights that in the KurSL model amplitude of each oscillator is strictly connected to, and can be expressed in terms of, phase dynamics. With such significant simplification, one only needs to solve phase dynamics (12) to obtained time series for a set of oscillators.

In case of most expanded coupling function $C(\vec{\phi}, t)$ (11) oscillatory solution will have a form of

$$
y_{i}(t)=\frac{r_{i 0} \sqrt{\dot{\omega}_{i 0}} \cos (\phi(t))}{\sqrt{\omega_{i}+\sum_{m=1}^{M} \sum_{j=1}^{N} k_{i j}^{m} \sin \left(m\left(\phi_{j}-\phi_{i}\right)\right)}},
$$

which depends on order $M$, all of the initial values, coupling strengths $k_{i j}^{m}$ between all the oscillators. Such definition means, that the model of an order $M, K u r S L_{M}$, incorporates all possible models up to its order. Transition from order $M$ to $M^{\prime}<$ $M$ is performed by setting all intermediate coupling factors $k^{m}=0$, where $m \in$ $\left(M^{\prime}, M\right]$. It follows that a solution space of $M^{\text {th }}$ order model, $\mathcal{S}_{M}$, is a subspace of solution space of any higher order model, $\mathcal{S}_{M} \subset \mathcal{S}_{M+m} \forall m \in \mathbb{N}$. 


\subsection{Examples}

Model's high complexity makes it difficult to be analysed analytically. In this section, a few special cases of the KurSL model evaluation are presented, highlighting some of its unique properties.

\section{No coupling}

The simplest form of the KurSL, yet of vital importance, is a system with noninteracting oscillators. In such a case oscillators can be treated as if they were isolated and had couplings factors for all pairs $k_{i j}=0$. This assumption affects the model to zero all but the intrinsic frequency terms in the Kuramoto coupling. With the instantaneous frequency being constant and equal $\dot{\phi}_{i}=\omega_{i}$ the solutions for the KurSL are equal to the Sturm-Liouville eigenproblem under $Q_{i}(t)=\omega_{i}^{2}$. Solutions to such stated problem are harmonic oscillators with the frequency being intrinsic frequency $y_{i}(t)=r_{0} \sin \left(\omega_{i} t+\theta\right)$. Such result is expected since when there is no interaction between harmonic oscillators they should preserve their harmonic nature.

\section{Complex oscillations}

As previously mentioned, the complexity of solution depends heavily on the number of oscillators and the order of the model. To illustrate the behaviour of the model representing complex oscillations, we describe a case with four oscillators generated using KurSL of order 3. The exact parameters used to synthesise components are presented in Table 1. In this table, intrinsic frequency and both initial phase and amplitude are denoted as previously in the article. Columns indicated by $k_{i j}^{m}$ refer to the order $m$ of the coupling with indices $i$ and $j$ referring to pairs of oscillators. Figure 2 contains all generated components presented individually and their sum is presented in Figure 3. In both figures, the left panel holds representations in the time domain with the series presented in blue and in case of individual oscillators the red colour indicates their instantaneous amplitudes. In contrast to harmonic oscillators, these components display non-constant amplitude with visible changes in frequency. These characteristics are also visible in the Fourier spectra that are presented in the right panel of Figure 2. One can see that all components have few small peaks close to one dominant peak that corresponds to the respective intrinsic frequency. These satellite peaks are a result of the coupling, and an underlying model characterises their properties. Despite the difficulty in describing them analytically, it has been observed that there are typically two additional peaks for each pair of oscillators with their positions and amplitudes dependent on the relative values of intrinsic frequencies and coupling strengths. The order of the model can significantly influence these properties by increasing absolute coupling strength, although it has a smaller impact on the number of peaks. Further in-depth analysis through simulations is presented in the following section. 
Table 1: Parameters used to generate an example of the KurSL model. The simulation was performed assuming four oscillators each with coupling up to $3^{\text {rd }}$ order. Intrinsic frequency, initial phase and amplitude are denoted as $\omega, \theta$ and $r_{0}$, respectively. Values $k_{i j}^{m}$ indicate coupling strengths between respective oscillators as indicated by row and column ordinals for $m^{\text {th }}$ harmonic.

\begin{tabular}{|c|c|c|c|c|c|c|c|c|c|c|c|c|c|c|c|}
\hline \multirow[b]{2}{*}{$i$} & \multirow[b]{2}{*}{$\omega / 2 \pi$} & \multirow[b]{2}{*}{$\theta$} & \multirow[b]{2}{*}{$r_{0}$} & \multicolumn{4}{|c|}{$k_{i j}^{1}$} & \multicolumn{4}{|c|}{$k_{i j}^{2}$} & \multicolumn{4}{|c|}{$k_{i j}^{3}$} \\
\hline & & & & 1 & 2 & 3 & 4 & 1 & 2 & 3 & 4 & 1 & 2 & 3 & 4 \\
\hline 1 & 2 & 1.2 & 1.0 & & 2.0 & 0.5 & -1.2 & & 1.5 & 0.2 & -0.4 & & 0.5 & 1.8 & 0.2 \\
\hline 2 & 5 & 2.5 & 1.5 & -2.0 & & 3.5 & -3.3 & -7.0 & & 2.1 & 4.2 & 4.1 & & 1.2 & 1.2 \\
\hline 3 & 10 & 0.0 & 1.0 & 2.2 & 0.5 & & 1.7 & 3.2 & 0.3 & & 0.1 & 3.8 & -2.2 & & 6.0 \\
\hline 4 & 13 & 2.0 & 1.0 & 0.1 & 1.9 & 1.9 & & 10.0 & -2.1 & 10.7 & & 0.0 & 9.1 & -1.5 & \\
\hline
\end{tabular}
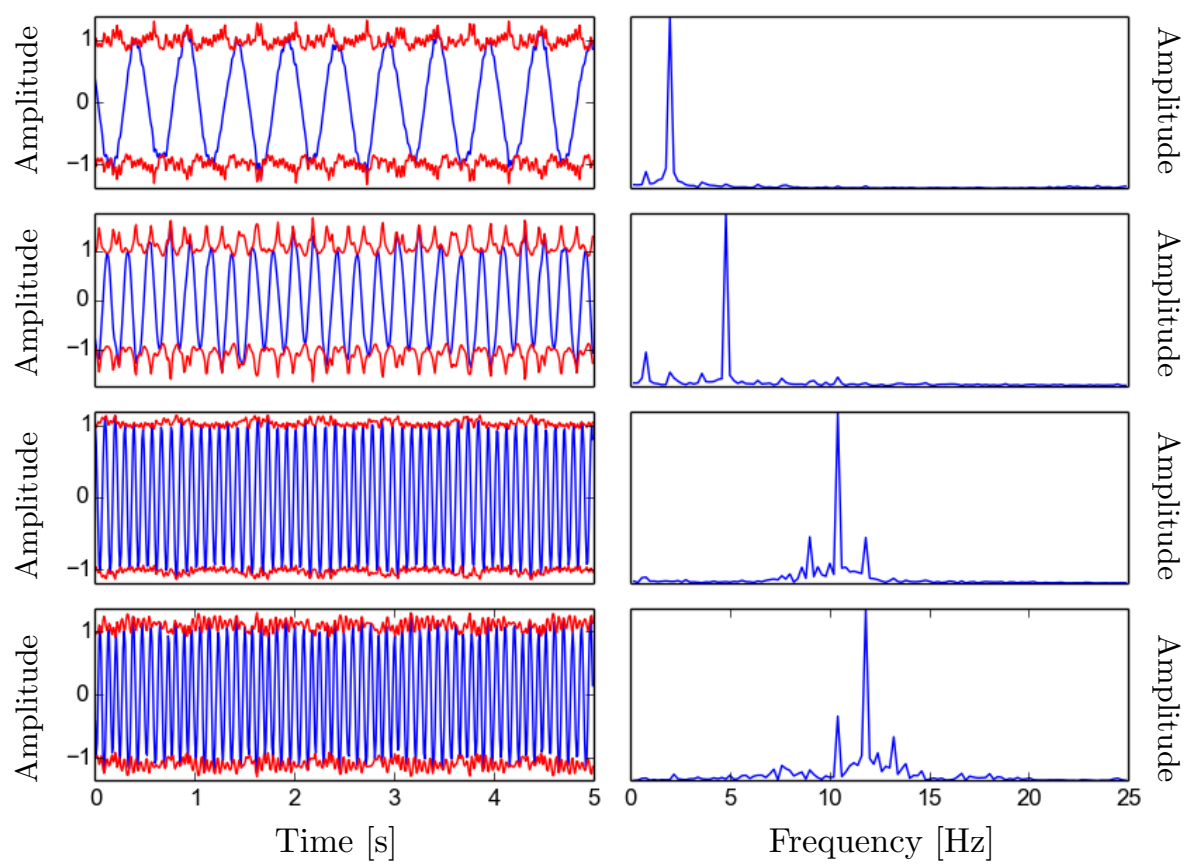

Fig. 2: Simulation on the KurSL model of order 3 assuming four oscillators and other parameters according to Table 1. The left column displays all components (blue) with their amplitudes in red, whereas the right-hand side has respective component's normalised Fourier transformation.

\section{Parameters mapping}

As noted previously, due to the high complexity of the model it is difficult to solve it analytically, when considering more than two oscillators. Nevertheless, analysis 


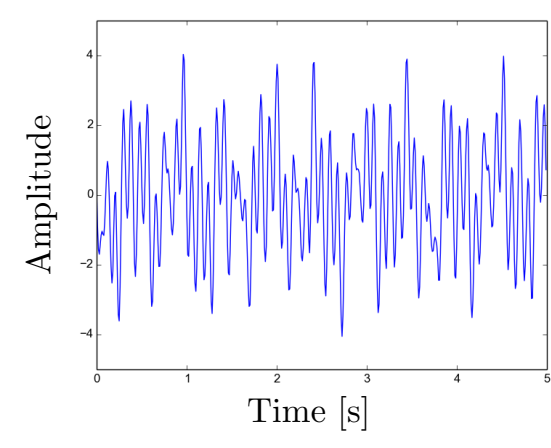

(a) Time domain

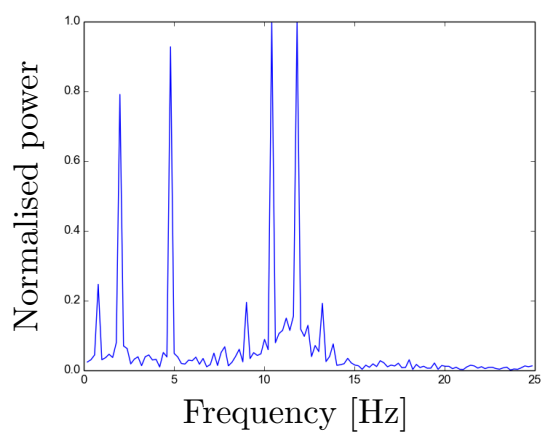

(b) Frequency domain

Fig. 3: A collective result in time and frequency domains of the generated signal using a model of order 3 with parameters from Table 1.

can be performed through numerical simulations. In this section, we investigate the behaviour of the mapping function from parameter space into time series space. This is done by traversing the parameter space and, for each configuration of the parameters, observing where these positions are mapped in the space of time series. By studying such behaviour, one can understand how changes to the parameters affect the shape of output time series. Knowing how a trajectory in one of the spaces is projected onto the other can often provide insights on how these spaces are shaped. Moreover, such traversing with small steps can be considered as an approximation for a partial derivative, which can be further utilised to analyse how the general outcome is affected by small changes in parameter space.

In this section, notation of $\mathcal{P}_{\mathcal{N}}$ and $\mathcal{S}_{\mathcal{N}}$ are used for parameter and time series spaces, respectively. The parameter space $\mathcal{P}_{\mathcal{N}}$ refers to space with all possible input parameters for the KurSL method. For this reason, each position $\mathbf{p}$ should be indexed with the number of oscillators and the order $M$ of the model, i.e. $\mathbf{p}_{\mathcal{N}, M}$. However, for the brevity, when referring to a particular selection from subspace, indices will be omitted as their values should be clear from the context. Such contraction will be denoted as

$$
\mathbf{p}_{x}:=\mathbf{p}_{\mathcal{N}, M ; x}=\left[\omega_{1}, \ldots, \omega_{\mathcal{N}}, r_{01}, \ldots, r_{0 \mathcal{N}}, \theta_{1}, \ldots, \theta_{\mathcal{N}}, k_{1,1}^{1}, \ldots, k_{2, \mathcal{N}}^{1}, \ldots, k_{\mathcal{N}, \mathcal{N}}^{M}\right]
$$

where the parameter $\mathbf{p}$ vector has $N=\mathcal{N}(3+M(\mathcal{N}-1))$ values. Assuming a Euclidean norm in these spaces means that for two vectors $\mathbf{p}_{1}$ and $\mathbf{p}_{2}$ the distance between them is given as

$$
\mathcal{M}_{\mathcal{P}}\left(\mathbf{p}_{x}, \mathbf{p}_{y}\right)=\sqrt{\sum_{i=1}^{n}\left(p_{x_{i}}-p_{y_{i}}\right)^{2}}
$$


Table 2: Initial parameters used for all experiments in this section. In each experiment, a single parameter was chosen and modified accordingly.

\begin{tabular}{c|ccc|cccc}
$n$ & $\omega / 2 \pi$ & $\theta$ & $r_{0}$ & \multicolumn{5}{|c}{$k_{i j}$} \\
& & & & 1 & 2 & 3 & 4 \\
\hline 1 & 30 & $\pi$ & 2 & & 0.1 & 2.2 & 4.2 \\
2 & 25 & 0 & 3 & 1.1 & & 2.0 & 1.1 \\
3 & 17 & 0 & 5 & 0.2 & 2.2 & & -0.9 \\
4 & 10 & 2 & 1 & 0.1 & 0.1 & 0 &
\end{tabular}

with $x$ and $y$ being all corresponding parameters for vectors $\mathbf{p}_{x}$ and $\mathbf{p}_{y}$, respectively. For example, if two vectors differ only at $\theta_{i}$ value by $\Delta \theta_{i}$, the distance will be $\mathcal{M}_{\mathcal{P}}\left(\mathbf{p}_{x}, \mathbf{p}_{y}\right)=\left|\Delta \theta_{i}\right|$.

An appropriate mathematical structure for representing time series, in general, would require defining a space over continuous functions. However, since all signals are recorded and discretised, this means one can utilise similar metric as in parameters space. Each oscillation can be stored in an array of a length $T_{N}$ and since there are $\mathcal{N}$ oscillations in reconstruction, the whole time series vector $\mathcal{S}$ can be described as

$$
\mathbf{s}=\left[s_{1}\left(t_{0}\right), \ldots, s_{1}\left(t_{T_{N}}\right), s_{2}\left(t_{0}\right), \ldots s_{2}\left(t_{T_{N}}\right), \ldots s_{\mathcal{N}}\left(t_{T_{N}}\right)\right]
$$

This means that all oscillatory components were concatenated to create a single vector of length $\mathcal{N} \times T_{N}$. It also means that the distance between two vectors $\mathbf{s}_{1}$ and $\mathbf{s}_{2}$ is

$$
\mathcal{M}_{\mathcal{S}}\left(\mathbf{s}_{1}, \mathbf{s}_{2}\right)=\sqrt{\sum_{i=1}^{n}\left(s_{x_{i}}-s_{y_{i}}\right)^{2}},
$$

where, again, $x$ and $y$ are values corresponding to vectors $\mathbf{s}_{1}$ and $\mathbf{s}_{2}$.

An illustration of a mapping function $\mathcal{G}$ from the parameter space $\mathcal{P}$ onto the time series space $\mathcal{S}$ is presented in Figure 4. It highlights that a straight line in one space may not necessarily map onto a straight line in the other. Not only the curvature can differ between spaces, but also the distance between consecutive points can vary. Such behaviour indicates non-linearity of the mapping function. In order to present how each parameter affects the model and whether the KurSL is a non-linear model, a series of experiments were performed. In each experiment, all parameters except for one were kept constant and for each position of the free parameter, a time series and phase dynamics were generated using the KurSL model. The core parameters used in all experiments are presented in Table 2. Although the number of oscillators was chosen to be $\mathcal{N}=4$, obtained results can be generalised. 


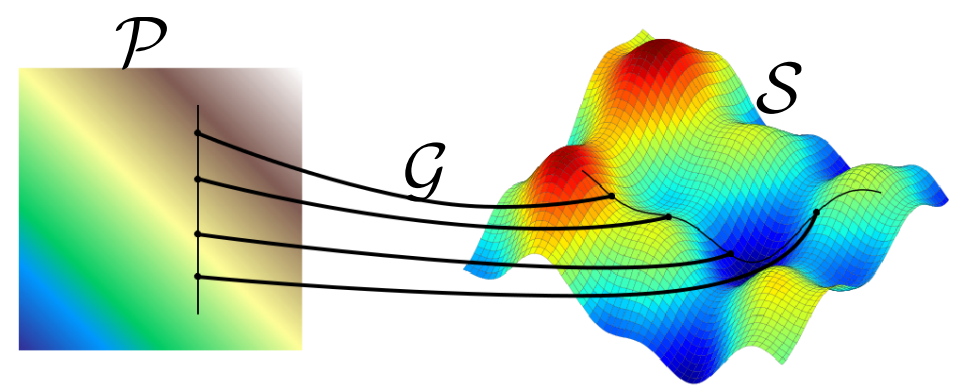

Fig. 4: A graphical representation of a mapping $\mathcal{G}$ between the parameter space $\mathcal{P}$ and the time series space $\mathcal{S}$. The function $\mathcal{G}$ maps points (dots) in parameter space $\mathcal{P}$ onto respective positions in time series space $\mathcal{S}$.

\section{Amplitude}

In the first experiment, the parameter of interest was amplitude. All parameters were initially set according to the Table 2 . Then, the amplitude of the $2^{\text {nd }}$ oscillator was modified in range of $r_{2} \in[1,10]$ with step $\Delta r=0.2$. Obtained results in the form of time series and phase dynamics are cumulatively presented in Figure 5a and $5 \mathrm{~b}$, respectively. These graphs display changes in the amplitude of time series or instantaneous frequency depending on the value of the parameter. Each row represents a different oscillator with the horizontal and vertical axes corresponding to time and component's amplitude values, respectively. Both figures are colourcoded with legends on the side showing their numerical values.

As it can be seen from these graphs, all but one plots have the same dynamics for each value of the variable. The exception is obtained for the time series in the second oscillator. Such result is due to the amplitude $r_{i}$ manifesting itself only as a simple scaling value in the KurSL model (Eq. 21). Such behaviour is additionally expected to produce monotonous mapping function from $\mathcal{P}$ into $\mathcal{S}$ space. Indeed, this can be observed in Figure 6, where the distances in time series space $\mathcal{S}$ are presented. The top graph shows the absolute distance, i.e. $\left\|\mathbf{s}_{\mathbf{p}}\right\|$, whereas the bottom shows relative distance to the previous parameter $\mathbf{p}$, i.e. $\mathcal{M}_{\mathcal{S}}\left(\mathbf{s}_{\mathbf{p}_{i}}, \mathbf{s}_{\mathbf{p}_{i+1}}\right)$. The behaviour of both functions can be explained by noticing that in this case, the metric function behaves like

$$
f(r)=\sqrt{a r^{2}+b},
$$

with respect to the amplitude $r$. Investigating its changes with a constant step $\Delta r$ one can see from Equation (26) that the result has the form of

$$
\mathcal{M}_{\mathcal{S}}\left(\mathbf{s}_{\mathbf{p}_{i}}, \mathbf{s}_{\mathbf{p}_{i+1}}\right)=\sqrt{a(\Delta r)^{2}+b},
$$

which is due to all other parameters being constant and independent from amplitude $r$. This means that in this case expression $\mathcal{M}_{\mathcal{S}}\left(\mathbf{s}_{\mathbf{p}_{i}}, \mathbf{s}_{\mathbf{p}_{i+1}}\right)$ is constant. 


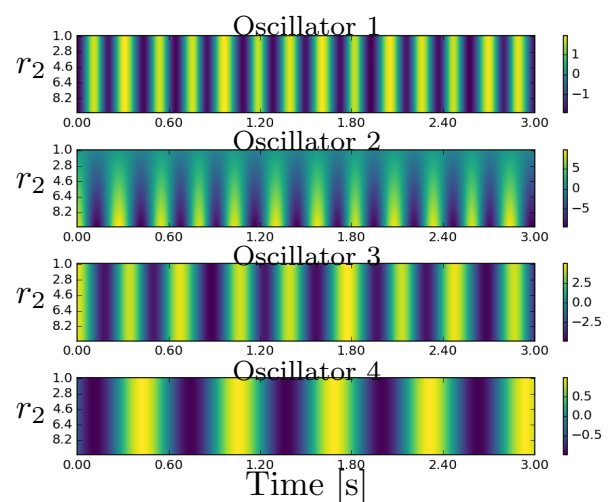

(a) Time series

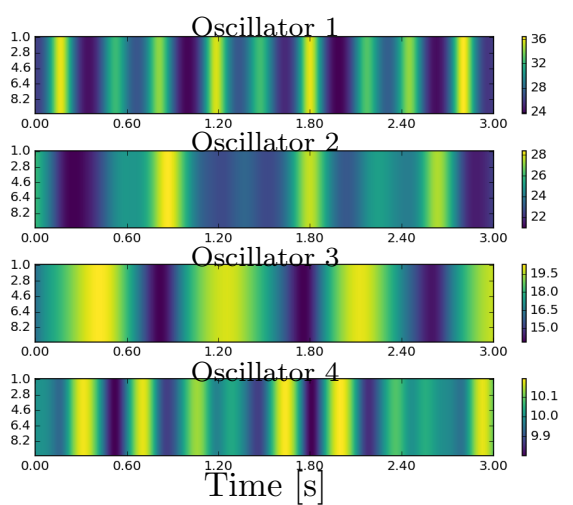

(b) Instantaneous frequency

Fig. 5: Colour-coded representation of time series obtained when modifying amplitude parameter, $r_{2}$. Each graph corresponds to a different oscillator (initial values Tab. 2). Horizontal and vertical axes correspond to time and $r_{2}$ values, respectively.
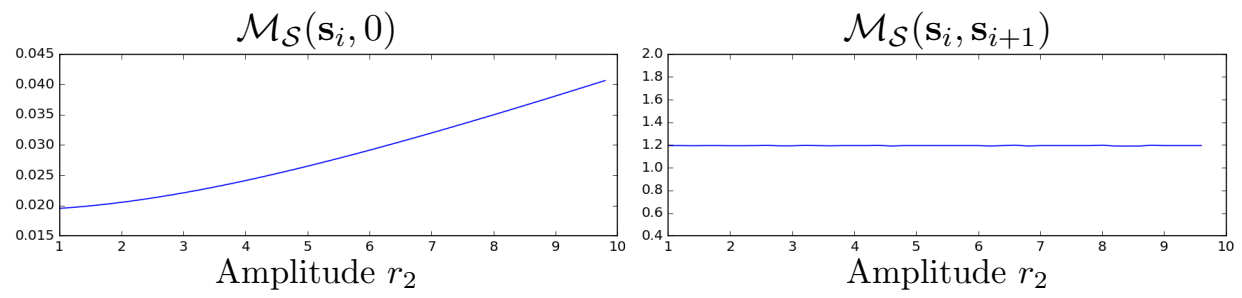

Fig. 6: Distance values of time series as a function of amplitude parameter, $r_{2}$. The left plot displays the absolute distance of the vector $\left\|\mathbf{s}_{\mathbf{p}}\right\|$, whereas the right one is a distance to the previous vector $\mathbf{p}$ in parameter space, i.e. $\mathcal{M}_{\mathcal{S}}\left(\mathbf{s}_{\mathbf{p}_{i}}, \mathbf{s}_{\mathbf{p}_{i+1}}\right)$.

\section{Initial phase}

The second experiment tested the behaviour when changing initial phase. Similarly to the previous experiment, all parameters have been kept constant except for one. The parameter has been modified in a range of $\theta_{2} \in[0,2 \pi]$ with a step $\Delta \phi=0.1$. All obtained signals are presented in Figure $7 \mathrm{a}$ and $7 \mathrm{~b}$, which displays the same information as Figure $5 \mathrm{a}$ and $5 \mathrm{~b}$, respectively, with the difference that vertical axis now corresponds to the initial phase $\theta_{2}$ values.

In this example, the effects of modulation are much more pronounced. Apparent effect is a shift in phase of the whole second oscillator, which directly corresponds to the parameter. The shift is not monotonous; when phase $\theta_{2} \approx 1.5$ there is a change in the progression. Due to the strong coupling between the second and the third 


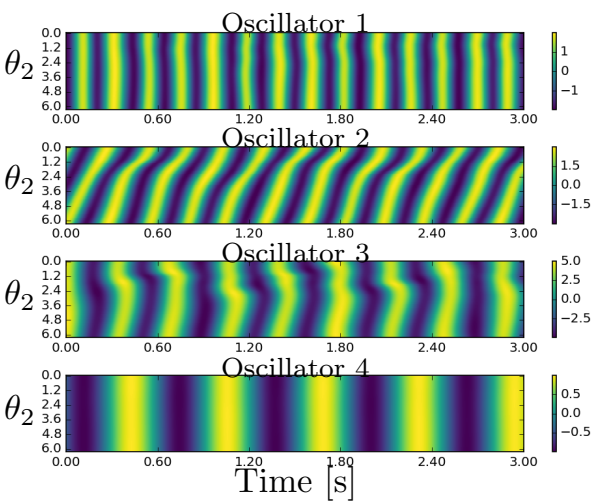

(a) Time series

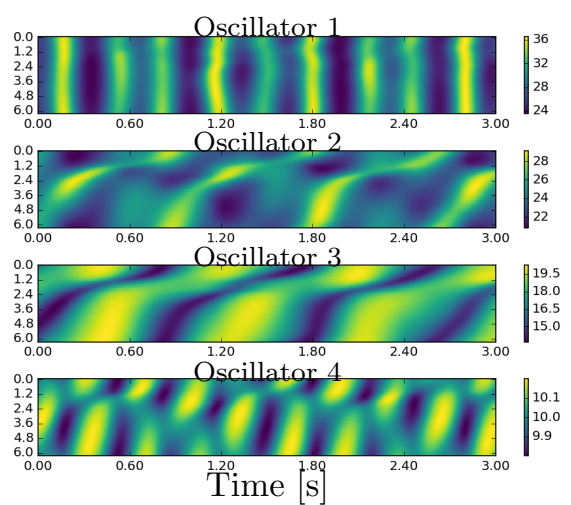

(b) Instantaneous frequency

Fig. 7: Colour-coded representation of time-series obtained when modifying phase parameter, $\phi_{02}$. Each graph corresponds to a different oscillator (initial values Tab. 2). Horizontal and vertical axes correspond to time and $\phi_{02}$ values, respectively.
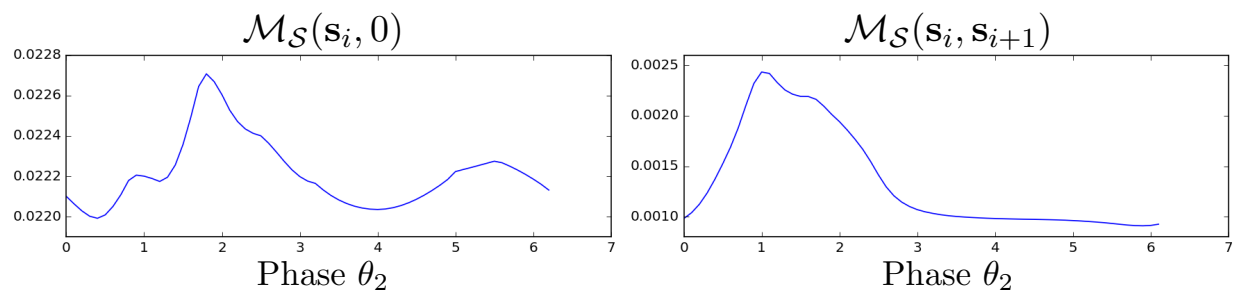

Fig. 8: Distance values of time series as a function of phase parameter, $\phi_{02}$. The left plot displays the absolute distance of the vector $\left\|\mathbf{s}_{\mathbf{p}}\right\|$, whereas the right one is a distance to the previous vector $\mathbf{p}$ in parameter space, i.e. $\mathcal{M}_{\mathcal{S}}\left(\mathbf{s}_{\mathbf{p}_{i}}, \mathbf{s}_{\mathbf{p}_{i+1}}\right)$.

oscillators, this transition is also visible in the third oscillator and indirectly in the first. Effects in the fourth row are unnoticeable in time series. In the phase dynamics, however, they seem to introduce a constant shift which repeats itself every $1.2 \mathrm{~s}$. This effect is more comprehensible when analysing distance graphs presented in Figure 8. Both distance metrics peaks are close to $\theta_{2} \approx 1.5$. This means that while preserving constant change in phase parameter, there is an increase followed by decrease in distance values. Observing the crests one can see that these modulations have no significant affect on frequency, although their widths and shape can differ (Fig. 7a). 


\section{Coupling factor}

In another experiment, the coupling strength between the first and the second oscillator has been modulated. Again, all values were kept constant (Tab. 2) except for $k_{21}$, which modulation range was set to $[-6,6]$, with step $\Delta k=0.1$. Collective results and distance values are presented in Figures $9 \mathrm{a}, 9 \mathrm{~b}$ and 10. In this case, modulations in both frequency and amplitude are visible. On the first sight it seems that effects of $k$ modulations are symmetrical - the further from $k=0$ the more modulations on all components. This is especially pronounced in dynamics Figures $9 \mathrm{a}$ and $9 \mathrm{~b}$, as well as in the relative distance plot 10. It seems that there are two maxima for approximately $k=-5$ and $k=5$. When coupling value reaches these points, the frequency of the second oscillator is matching the frequency of the first one closely. With the increase of coupling strength $|k|>5$ the distance is decaying and stabilising. However, based on the actual shape of the peak in Fig. 10 one can see that there is a different behaviour depending on the sign of the coupling strength $k$. The signal for large positive $k$ converges faster to the final frequency.

It has to be pointed out, that although the modulated coupling is present between the first and the second components, the first component is not visibly affected. In comparison, the third oscillator seems to be affected more significantly. Such behaviour is explained by the effect of coupling not being normalised to the intrinsic frequency. From equation (12) it seems clear that the bigger intrinsic frequency, the bigger coupling strength has to be to affect phase modulations. Third component's $\omega_{3}$ is about two times smaller than the first component's. This difference leads to larger sensitivity in modulations, even if indirect. A similar result can be observed for the fourth component; despite having little variation in the time series, it has a relatively big impact on its instantaneous frequency dynamics.

\section{Intrinsic frequency}

The parameter that seems to have the most visible impact on both time series and phase dynamics is the intrinsic frequency, $\omega$. In the fourth experiment, the frequency of the second component $\omega_{2}$ has been varied in the range [5,45], with step $\Delta \omega=0.1$. Such modulations display clear patterns that can be observed in colour-coded dynamics of time series (Fig. 11a) and phase dynamics (Fig. 11b). In both figures, all components have been visibly affected. Observed distances in Figure 12 show a major distortion in areas of $\omega \approx 30$ for the first, $\omega \approx 8$ for the fourth oscillator and range $[13,22]$ for the second oscillator. These values seem to correspond directly to the frequency of other components, suggesting that there is resonance effect between oscillators. Sudden changes can be observed also when analysing distance plots in Fig. 12. In regions close to the frequency of any other oscillator there are dynamical changes. However, in regions relatively far, i.e. when $\omega \approx 15$ and $\omega>33$, changes in metric values are more gradual. Interesting pattern can also be observed in the fourth oscillator's phase dynamics. Despite having a relatively constant position of peaks, their amplitudes are modulated in pattern 


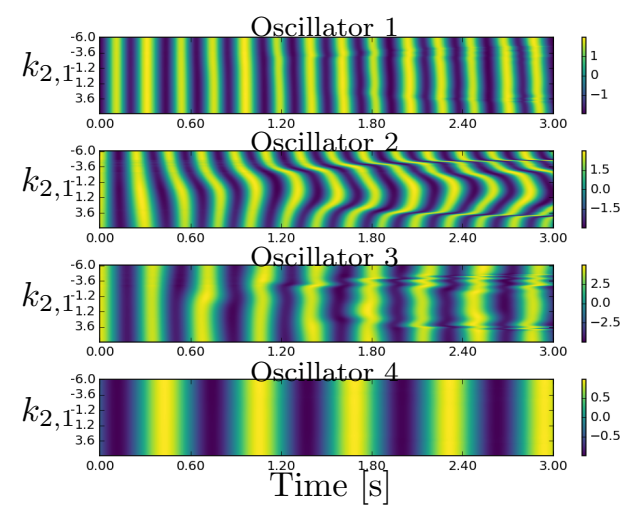

(a) Time series

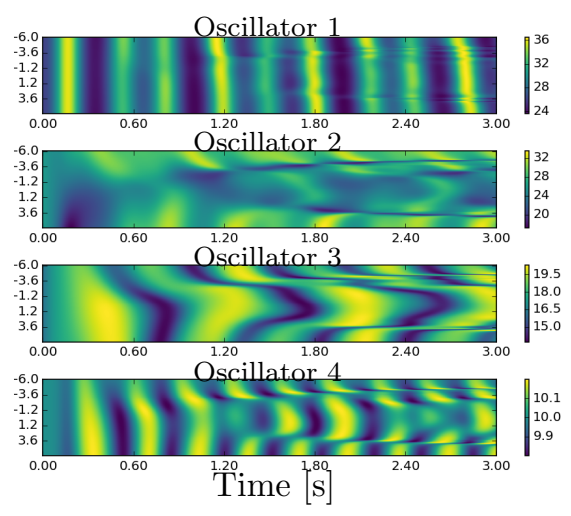

(b) Instantaneous frequency

Fig. 9: Set of components obtained by varying coupling strength $k_{2,1}$, i.e. between the first and the second components. The range of changes is from -6 to 6 with step $\Delta k=0.1$. Each graph represents the amplitude of the $n^{\text {th }}$ oscillator. The horizontal axis is the time and the vertical axis are the values of the coupling, $k_{2,1}$.
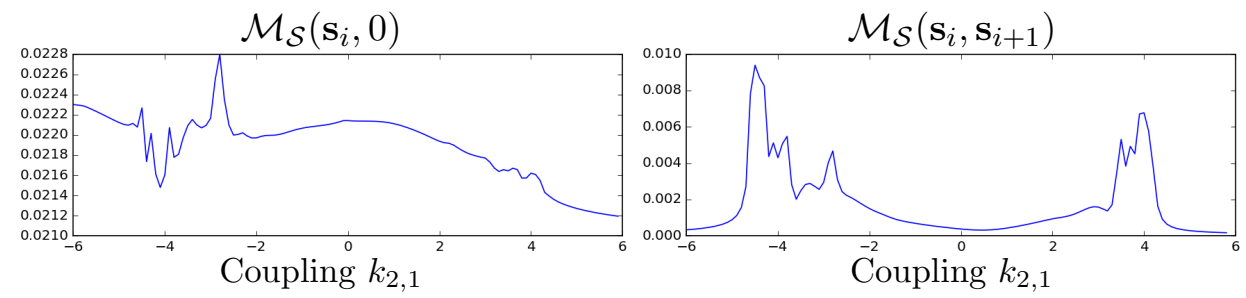

Fig. 10: Distance values of time series as a function of intrinsic frequency parameter, $k_{2,1}$. Left plot displays the absolute distance of the vector, whereas the right one is distance to the previous vector $\mathbf{p}$ in parameter space.

visible for the third oscillator.

\section{Summary of experiments}

All these experiments allow for descriptive sensitivity analysis which provides insights into the model's behaviour. By studying it, one can understand how changes in parameters affect the form of generated time series. Numerical evaluations on presented ranges made it possible to show changes in the mapping function. These can be treated as traversing along a specific path within time series space $\mathcal{S}$ and parameter space $\mathcal{P}$. Traversing along these trajectories allow for observing how the distance between consecutive points changes. In general, the mapping function be- 


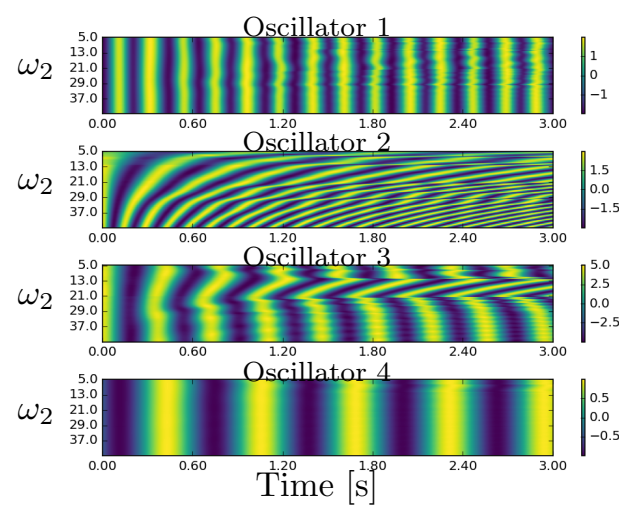

(a) Time series

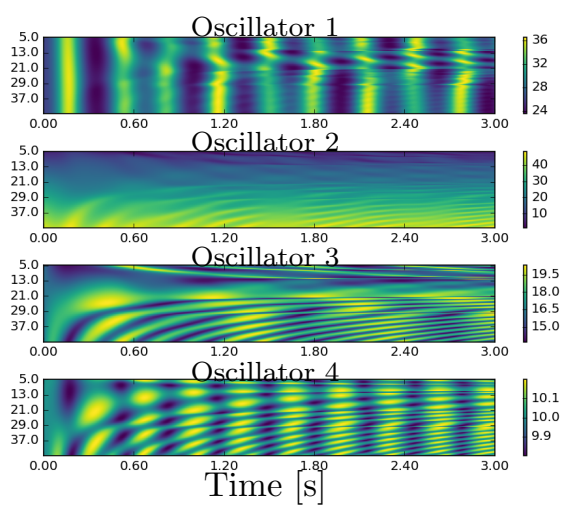

(b) Instantaneous frequency

Fig. 11: Colour-coded representation of time-series obtained when modifying intrinsic frequency parameter, $\omega_{2}$. Each graph corresponds to a different oscillator (initial values are in Tab. 2). The horizontal and vertical axes correspond to time and $\omega_{2}$ values, respectively.
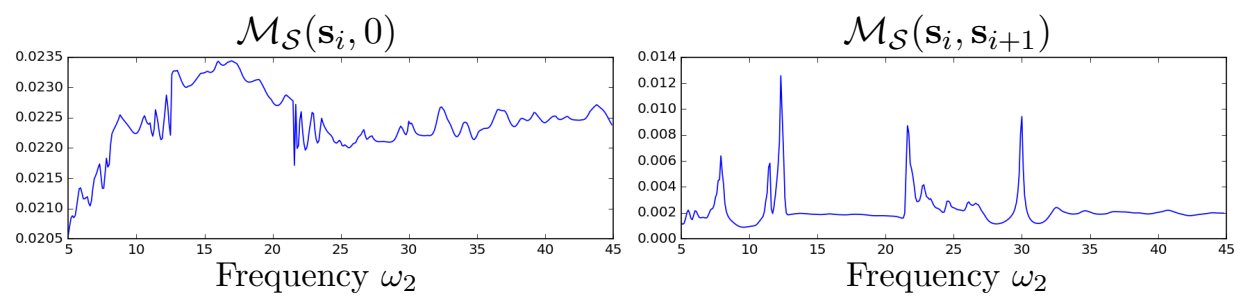

Fig. 12: Distance values of time series as a function of intrinsic frequency parameter, $\omega_{2}$. The left plot displays the absolute distance of the vector, whereas the right one is the distance to the previous vector $\mathbf{p}$ in parameter space.

tween mentioned spaces is non-linear. Out of presented examples, only traversing along the amplitude path did not modify the distance metric whereas the rest of parameters had a significant effect on the whole system. One can see that the directed gradient changes non-monotonically and the level of variation depends on the position from which the step was made. In some regions, these variations are substantially different than in others, even when perturbing by the same amount, but despite occasional sharp changes, there is no reason to suspect that the gradient is not continuous. It is expected that these sudden changes can depend on relative values of all parameters, highlighting possible interaction effects between oscillators, such as frequency alignment. 


\section{Conclusions}

The paper introduced a novel model, KurSL, which can capture behaviour of complex signals generated by systems of mutually interacting oscillations. The significance of this model stems from its ability to extract phase dynamics of a set of coupled oscillators directly from recorded time series. Such analysis is typically obtained by performing bandpass filtering [Andrade et al., 2008; Stankovski et al., 2015], or by assuming a specific form of the response [Busch et al., 2009], whereas KurSL uses the general form of oscillations. This generalisation has been achieved through a combination of the Sturm-Liouville oscillation model and the Kuramoto coupling model. Integration of the two approaches builds on their strengths. The former model describes an individual oscillation and its properties, whereas the latter focuses on capturing interactions between several oscillators. These intend to reflect interacting behaviour present in most physical systems, where each oscillator affects and depends on other elements. Such description leads to the production of components as general oscillations with variable amplitude and frequency. In cases when there are no interactions between components, KurSL will simply describe these as composed of harmonic oscillators, as would be done by the Fourier analysis. This encapsulation makes the KurSL model particularly suitable for explaining a wide range of oscillating phenomena and processes. Thus, the model opens up possibilities to analyse data that are expected to reflect interacting oscillatory processes such as physiological data [de Lima et al., 2006], brain signals [Sweeney-Reed and Nasuto, 2009], atmospheric data [Huang et al., 1998] or financial events [Lux and Marchesi, 1999].

Introduction of a new model opens many possible research avenues. In Section 4 we examined the behaviour of the mapping from the KurSL's parameter space to the time series and phase dynamics spaces. The investigation has been performed by manipulating individual parameters within certain ranges, while keeping other parameters fixed. This procedure approximates calculation of the scaled partial derivatives along specified axes. This analysis revealed nontrivial dependency of the obtained decompositions on the parameters. Only the mapping of the amplitude is monotonically increasing, which was additionally verified analytically. In case of the other parameters, effects of modulations have been observed in all oscillators confirming the non-linear behaviour of the model.

The behaviour observed while manipulating the coupling factor was interesting and not intuitive. All effects depend greatly on the strength of the interaction between investigated component and the others. There was a visible difference in distance metric when comparing response produced with positive and negative values $k$ (Fig. 10). The sign in the coupling factor indicates, whether the oscillators are stabilising the system by pulling towards each other, or destabilising it by pushing away in the phase domain. It seems that in the presented example investigated interaction had little effect on global state, until a particular absolute value of the coupling $k$ was reached. The mechanisms behind this phenomenon will be a subject 
of future study.

One of the observed effects taking place in the phase domain is likely due to resonance. When the frequency parameter was modified for some $\omega_{2}$ values, there was a visible sharp peak in the distance between consecutive points in time series space. For example, this is visible for $\omega_{2} \approx 30$, which is equal to the first oscillator's intrinsic frequency $\omega_{1}$. The expectation is that this effect is additionally affected by the coupling strength $k$ between oscillators under consideration.

The KurSL is capable of representing complex structures that may be present in the data due to convoluted interactions of the oscillators which generate them. Future work will focus on introducing an automated KurSL parameters' estimation method from a given empirical time series that will effectively amount to the identification of the number of the oscillators and estimation of the resultant model parameters (parameter estimation). Such task is non-trivial due to high computational complexity and non-linearity of the model; it is difficult to define efficient cost function with convex behaviour. This forces search method to use heuristic approach using some of the domain knowledge. Nevertheless, projecting signal onto the model results in a decomposition of the input signal into the constituent oscillations most consistent with the data and the model constraints. Such decomposition allows then to quantitatively and qualitatively describe oscillatory phenomena within the analysed system. Implementation of the current KurSL forward model is available to download from author's webpage [Laszuk, 2014].

\section{References}

Acebrón, J. A., Bonilla, L. L., Pérez Vicente, C. J., Ritort, F. and Spigler, R. (2005). The Kuramoto model: A simple paradigm for synchronization phenomena. Reviews of Modern Physics, 77: 137-185.

Andrade, A. O., Kyberd, P. and Nasuto, S. J. (2008). The application of the Hilbert spectrum to the analysis of electromyographic signals. Information Sciences, 178: $2176-2193$.

Andrade, A. O., Nasuto, S., Kyberd, P., Sweeney-Reed, C. M. and Kanijn, F. R. V. (2006). EMG signal filtering based on Empirical Mode Decomposition. Biomedical Signal Processing and Control, 1: 44-55.

Boashash, B. (1992). Estimating and interpreting the instantaneous frequency of a signal. i. fundamentals. Proceedings of the IEEE, 80: 520-538, doi:10.1109/5. 135376.

Breakspear, M., Heitmann, S. and Daffertshofer, A. (2010). Generative models of cortical oscillations: Neurobiological implications of the kuramoto model. Frontiers in Human Neuroscience, 4: 190.

Brown, E., Holmes, P. and Moehlis, J. (2003). Globally coupled oscillator networks. Perspectives and problems in nonlinear science: A celebratory volume in honor of Larry Sirovich: 183-215.

Busch, N. a., Dubois, J. and VanRullen, R. (2009). The phase of ongoing EEG 
oscillations predicts visual perception. The Journal of neuroscience : the official journal of the Society for Neuroscience, 29: 7869-76.

Carvalhaes, C. and de Barros, J. A. (2015). The surface laplacian technique in EEG: Theory and methods. International Journal of Psychophysiology, 97: 174-188.

Chu, P. C., Fan, C. and Huang, N. E. (2013). Derivative-optimized empirical mode decomposition for the HilbertHuang transform. Journal of Computational and Applied Mathematics.

Cohen, L. (1995). Time-frequency Analysis, reprint ed., Prentice Hall PTR, ISBN 9780135945322.

Cohen, M. X. and Gulbinaite, R. (2013). Five methodological challenges in cognitive electrophysiology. NeuroImage.

de Lima, E. R., Andrade, A. O., Pons, J. L., Kyberd, P. and Nasuto, S. J. (2006). Empirical mode decomposition: a novel technique for the study of tremor time series. Medical and Biological Engineering and Computing, 44: 569-582.

Duggento, A., Stankovski, T., McClintock, P. V. E. and Stefanovska, A. (2012). Dynamical Bayesian inference of time-evolving interactions: From a pair of coupled oscillators to networks of oscillators. Physical Review E - Statistical, Nonlinear, and Soft Matter Physics, 86: 1-16.

Freiling, G. and Yurko, V. A. (2001). Inverse Sturm-Liouville problems and their applications, NOVA Science Publishers New York, ISBN 9781560729099.

Galán, R. F., Ermentrout, G. B. and Urban, N. N. (2005). Efficient estimation of phase-resetting curves in real neurons and its significance for neural-network modeling. Physical review letters, 94: 158101.

Galka, A. (2000). Topics in Nonlinear Time Series Analysis: With Implications for EEG Analysis, World Scientific, ISBN 978-981-02-4148-3.

Gou, K. and Chen, Z. (2015). Inverse Sturm-Liouville Problems and their Biomedical Engineering Applications. JSM Mathematics and Statistics, 2: 1-9.

Halliday, D., Resnick, R. and Walker, J. (2000). Fundamentals of Physics, 6th ed., Wiley, ISBN 0471332364.

Herzog, E. D. (2007). Neurons and networks in daily rhythms. Nature reviews. Neuroscience, 8: 790-802.

Huang, N. E., Shen, Z., Long, S. R., Wu, M. C., Shih, H. H., Zheng, Q., Yen, N.-C., Tung, C. C. and Liu, H. H. (1998). The empirical mode decomposition and the Hilbert spectrum for nonlinear and non-stationary time series analysis. Proceedings of the Royal Society A: Mathematical, Physical and Engineering Sciences, 454: 903-995.

Kuramoto, Y. (1975). Self-entrainment of a population of coupled non-linear oscillators, in International Symposium on Mathematical Problems in Theoretical Physics, Lecture Notes in Physics, Vol. 39, ed. H. Araki, Springer Berlin Heidelberg, ISBN 978-3-540-07174-7, pp. 420-422.

Landau, L. D. and Lifshitz, E. M. (1965). Quantum mechanics, Non-relativistic Theory, Pergamon Press. 
Laszuk, D. (2014). Python implemention of Empirical Mode Decomposition algorithm. http://www. laszukdawid.com/codes.

Laszuk, D., Cadenas, O. and Nasuto, S. J. (2016). On the Phase Coupling of Two Components Mixing in Empirical Mode Decomposition. Advances in Adaptive Data Analysis, 8: 1650004-1-1650004-11.

Lux, T. and Marchesi, M. (1999). Scaling and criticality in a stochastic multi-agent model of a financial market. Nature, 397: 498-500.

Michaels, D. C., Matyas, E. P. and Jalife, J. (1987). Mechanisms of sinoatrial pacemaker synchronization: a new hypothesis. Circulation research, 61: 704-714.

Molla, M. K. I., Tanaka, T., Rutkowski, T. M. and Cichocki, A. (2010). Separation of EOG artifacts from EEG signals using bivariate EMD, in 2010 IEEE International Conference on Acoustics, Speech and Signal Processing, IEEE, ISBN 978-1-4244-4295-9, pp. 562-565.

Moran, R., Kiebel, S., Stephan, K., Reilly, R., Daunizeau, J. and Friston, K. (2007). A neural mass model of spectral responses in electrophysiology. NeuroImage, 37: 706-720.

Osterhage, H., Mormann, F., Wagner, T. and Lehnertz, K. (2008). Detecting directional coupling in the human epileptic brain: Limitations and potential pitfalls. Phys. Rev. E, 77: 011914, doi:10.1103/PhysRevE.77.011914.

Pontrelli, G. and de Monte, F. (2009). Modeling of mass dynamics in arterial drugeluting stents. Journal of Porous Media, 12: 19-28.

Rodrigues, F. A., Peron, T. K. D., Ji, P. and Kurths, J. (2016). The kuramoto model in complex networks. Physics Reports, 610: 1 - 98, the Kuramoto model in complex networks.

Rosenblum, M. G., Cimponeriu, L., Bezerianos, A., Patzak, A. and Mrowka, R. (2002). Identification of coupling direction: Application to cardiorespiratory interaction. Phys. Rev. E, 65: 041909, doi:10.1103/PhysRevE.65.041909.

Rutkowski, T. M., Mandic, D. P., Cichocki, A. and Przybyszewski, A. W. (2010). EMD approach to multichannel EEG data the amplitude and phase components clustering analysis. Journal of Circuits, Systems and Computers, 19: 215-229.

Sadilek, M. and Thurner, S. (2015). Physiologically motivated multiplex kuramoto model describes phase diagram of cortical activity. Scientific reports, $\mathbf{5}$.

Schnitzler, A. and Gross, J. (2005). Normal and pathological oscillatory communication in the brain. Nature reviews neuroscience, 6: 285.

Schrödinger, E. (1926). An undulatory theory of the mechanics of atoms and molecules. Phys. Rev., 28: 1049-1070.

Sharpley, R. C. and Vatchev, V. (2006). Analysis of the Intrinsic Mode Functions. Constructive Approximation, 24: 17-47.

Singer, A. (2006). Spectral independent component analysis. Applied and Computational Harmonic Analysis, 21: 135 - 144, special Issue: Diffusion Maps and Wavelets.

Stankovski, T., Ticcinelli, V., McClintock, P. V. E. and Stefanovska, A. (2015). 
Coupling functions in networks of oscillators. New Journal of Physics, 17: 035002. Strogatz, S. H. (1994). Nonlinear Dynamics and Chaos: With Applications to Physics, Biology, Chemistry, and Engineering.

Strogatz, S. H. (2000). From Kuramoto to Crawford: exploring the onset of synchronization in populations of coupled oscillators. Physica D: Nonlinear Phenomena, 143: $1-20$.

Sturm, J. C. F. (1836). Mémoire sur les équations différentielles linéaires du second ordre. Journal de mathématiques pures et appliquées, 1: 106-186.

Sweeney, K. T., McLoone, S. F. and Ward, T. E. (2013). The use of ensemble empirical mode decomposition with canonical correlation analysis as a novel artifact removal technique. IEEE transactions on bio-medical engineering, 60: 97-105.

Sweeney-Reed, C. M. and Nasuto, S. J. (2007). A novel approach to the detection of synchronisation in EEG based on empirical mode decomposition. Journal of computational neuroscience, 23: 79-111.

Sweeney-Reed, C. M. and Nasuto, S. J. (2009). Detection of neural correlates of selfpaced motor activity using empirical mode decomposition phase locking analysis. Journal of neuroscience methods, 184: 54-70.

Tauro, C. B., Tamarit, F. A., Gleiser, P. M. et al. (2014). Modeling spatial patterns in the visual cortex. Physical Review E, 90: 042818.

Teschl, G. (2012). Ordinary Differential Equations and Dynamical Systems, ISBN 978-0-8218-8328-0.

Wagner, T., Axmacher, N., Lehnertz, K., Elger, C. E. and Fell, J. (2010). Sleepdependent directional coupling between human neocortex and hippocampus. Cortex, 46: 256 - 263, doi:https://doi.org/10.1016/j.cortex.2009.05.012.

Winfree, A. T. (1967). Biological rhythms and the behavior of populations of coupled oscillators. Journal of Theoretical Biology, 16: 15-42. 